\title{
Chemical Sharpening, Shortening, and Unzipping of Boron Nitride Nanotubes
}

\author{
Yunlong Liao, Zhongfang Chen," John W. Connell,* Catharine C. Fay, Cheol Park, \\ Jae-Woo Kim, and Yi Lin*
}

\begin{abstract}
Boron nitride nanotubes (BNNTs), the one-dimensional member of the boron nitride nanostructure family, are generally accepted to be highly inert to oxidative treatments and can only be covalently modified by highly reactive species. Conversely, it is discovered that the BNNTs can be chemically dispersed and their morphology modified by a relatively mild method: simply sonicating the nanotubes in aqueous ammonia solution. The dispersed nanotubes are significantly corroded, with end-caps removed, tips sharpened, and walls thinned. The sonication treatment in aqueous ammonia solution also removes amorphous BN impurities and shortened BNNTs, resembling various oxidative treatments of carbon nanotubes. Importantly, the majority of BNNTs are at least partially longitudinally cut, or "unzipped". Entangled and freestanding BN nanoribbons (BNNRs), resulting from the unzipping, are found to be $\sim 5-20 \mathrm{~nm}$ in width and up to a few hundred nanometers in length. This is the first chemical method to obtain BNNRs from BNNT unzipping. This method is not derived from known carbon nanotube unzipping strategies, but is unique to BNNTs because the use of aqueous ammonia solutions specifically targets the B-N bond network. This study may pave the way for convenient processing of BNNTs, previously thought to be highly inert, toward controlling their dispersion, purity, lengths, and electronic properties.
\end{abstract}

\section{Introduction}

Boron nitride nanotubes (BNNTs), analogous in geometry with carbon nanotubes (CNTs), have exceptional mechanical, thermal, and optical properties..$^{[1-3]}$ These properties make BNNTs promising materials for many applications, such as polymeric composites ${ }^{[4]}$ and biological applications..$^{[5]}$ For example, BNNT-filled polymer composites are expected to have high mechanical strength and high thermal conductivity similar to those filled with CNTs, but in contrast are much lighter in color (usually white due to light scattering) and electrically insulating. ${ }^{[1-4]}$ These latter characteristics are attributed to the large bandgap $(\sim 6 \mathrm{eV})$ of BNNTs due to the less delocalized and polarized B-N bond network. BNNTs are also known to be very resistant to thermal and wet oxidations as well as many other chemical modifications.

In stark contrast, oxidation of CNTs has been proven very convenient and funda-

Y. Liao, Dr. J.-W. Kim, Dr. Y. Lin

National Institute of Aerospace

100 Exploration Way, Hampton

Virginia 23666-6147, USA

E-mail: yi.lin@nianet.org

Y. Liao, Prof. Z. Chen

Department of Chemistry

Department of Physics

University of Puerto Rico

Rio Piedras Campus, San Juan

Puerto Rico 00931, USA

E-mail: zhongfangchen@gmail.com

Dr. J. W. Connell, Dr. C. C. Fay, Dr. C. Park

NASA Langley Research Center

Hampton, Virginia 23681-2199, USA

E-mail: john.w.connell@nasa.gov

Dr. C. Park

Department of Mechanical and Aerospace Engineering

University of Virginia

Charlottesville, VA 22904, USA

Dr. Y. Lin

Department of Applied Science

The College of William and Mary

Williamsburg, VA 23185, USA

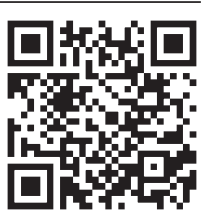

mentally important to modify the nanotube structure and morphology via controlled corrosive effects. ${ }^{[6-11]}$ For example, in the early explorations of the chemical properties of multi-walled CNTs (MWCNTs), it was found that air oxidation could lead to end-cap opening of the tubes accompanied by the nanotube tip sharpening and the tube wall thinning. ${ }^{[6,7]}$ Sonicating singlewalled CNTs (SWCNTs) or MWCNTs in strong oxidative acids, such as the mixture of sulfuric and nitric acids, led to nanotube shortening. ${ }^{[8-11]}$ Recently, oxidation-related treatments have been employed to successfully unzip MWCNTs into graphene nanoribbons (GNRs). ${ }^{[12-14]}$ For instance, Tour and co-workers extensively explored MWCNT unzipping using a potassium permanganate-sulfuric acid system. ${ }^{[12]}$ Dai and co-workers sonicated partially air-oxidized MWCNTs in a polymer solution to obtain GNRs. ${ }^{[14]}$

The equivalent corrosion and unzipping of BNNTs using chemical methods, however, has never been reported partially due to their strong resistance to oxidation and various chemical treatments. Compared to CNTs that usually start to decompose in air at $400-500{ }^{\circ} \mathrm{C}$, BNNTs usually remain stable to nearly $800{ }^{\circ} \mathrm{C} .{ }^{[15]}$ Thermal oxidation of BNNTs could only be carried out above this temperature when significant damage to the nanotubes might start to occur. ${ }^{[16 a]}$ Wet oxidation of BNNTs

DOI: 10.1002/adfm.201400599 
under relatively harsh conditions were also reported, ${ }^{[16 b]}$ in which hydrogen peroxide was used to react with the nanotubes at $120{ }^{\circ} \mathrm{C}$ for $24 \mathrm{~h}$ in an autoclave. Though BNNTs were found chemically active toward various Lewis bases, such as various amines and phosphines, ${ }^{[17-19]}$ the attachments of these functional groups have been considered mostly ionic in nature and seldom impart changes to the BNNT atomic structure. Reports are also available detailing the covalent functionalization of BNNTs by targeting the residual tube surface amino groups produced under nitrogen-rich conditions. ${ }^{[20,21]}$ It was suggested that such functionalization was sufficient to modify the bandgap of BNNTs. ${ }^{[22]}$ Direct covalent modification of B-N bond network was yet to be reported on BNNTs, but has very recently been achieved on boron nitride nanosheets (BNNSs), the planar relative of the nanotubes. ${ }^{[23,24]}$ Very reactive species such as nitrenes and oxygen radicals were used therein.

Only a handful of reports are currently available on cutting BNNTs in either circumferential (i.e., shortening) or longitudinal (i.e., unzipping) directions. For example, Zhi and coworkers pre-oxidized BNNTs at over $900{ }^{\circ} \mathrm{C}$ and sonicated the partially damaged nanotubes in solvents to obtain shortened tubes. ${ }^{[16 a]}$ Yap and co-workers were able to shorten BNNTs with noncovalent functionalities simply by sonicating the soluble nanotubes in aqueous solution under ambient conditions. ${ }^{[25]}$ The shortening was attributed to the high acoustic energy coupled with the individual dispersion of the nanotubes. Longitudinal unzipping of BNNTs yields boron nitride nanoribbons (BNNRs). The reported BNNT unzipping strategies include plasma etching ${ }^{[26]}$ and the expansion of intercalated potassium. ${ }^{[27]}$ These physical methods were first demonstrated with the unzipping of $\mathrm{CNTS}^{[28,29]}$ and subsequently applied to BNNTs. However, the known chemical unzipping methods for CNTs often involve oxidation, ${ }^{[12-14]}$ making them not directly applicable to BNNTs. To date, a chemical method that is specifically designed to achieve BNNT unzipping has yet to be reported.

In this article, it was demonstrated that the aqueous ammonia solution is an effective reagent to functionalize and disperse BNNTs with the assistance of low power sonication under ambient conditions. It is not suprising that ammonia, as a small Lewis base molecule, can be used for such purpose since similar chemistry is well known. ${ }^{[17-19]}$ However, unexpectedly but more importantly, the nanotube structures were significantly corroded. The observed corrosive effects included nanotube end-cap removal, tip sharpening, sidewall thinning, length shortening, and also partial or even full longitudinal unzipping. While BNNTs are inert to strong oxidative treatment conditions, their structural vulnerability to aqueous ammonia solutions opens up a pathway which may allow their convenient processing toward a variety of applications.

\section{Results and Discussion}

\subsection{Pristine BNNTs}

To date, techniques to grow BNNTs can be divided into two broad categories. ${ }^{[1-3]}$ The first category includes high temperature synthesis techniques, which involve vaporizing targets made of elemental boron or boron nitride, which reacts with nitrogen and condenses into BNNT solids. This method produces BNNTs with few defects and small numbers of walls but is limited to small batches (milligrams). ${ }^{[30,31]}$ The second category includes various low temperature synthesis methods, where the operating temperatures are typically between 600 and $1700{ }^{\circ} \mathrm{C}$, well below the vaporization temperature of pure boron (over $4000{ }^{\circ} \mathrm{C}$, depending on the applied pressure). These low temperature synthesis methods, including chemical vapor deposition (CVD), ${ }^{[32,33]}$ CNT substitution reaction, ${ }^{[34]}$ or ball-milling, ${ }^{[35]}$ are much more scalable and can routinely produce hundreds of milligrams to grams of BNNTs. Typical diameters of BNNTs from CVD and ball-milling are about an order of magnitude greater than those grown by high temperature synthesis method ( $\sim 50 \mathrm{~nm}$ vs. $\sim 5 \mathrm{~nm})$. Furthermore, these nanotubes often have comparably somewhat more defective morphologies due to the lower synthesis temperature.

The as-prepared pristine BNNT used in this study was made by laser-induced pressurized vapor condensation, a high temperature synthesis method, using only boron and nitrogen resource without any catalysts. ${ }^{[30]}$ The as-prepared pristine BNNTs consisted mostly of few-walled nanotubes that are of $\sim 2-10 \mathrm{~nm}$ in diameter. Single-walled and larger-diameter multiwalled nanotubes were also present but in lower amounts. These pristine nanotubes were long and heavily entangled (Figure 1a), with the tube ends only occasionally visible under transmission electron microscopy (TEM) imaging. The best estimation of their length was from a few to tens of $\mu \mathrm{m}$. At higher magnification (Figure 1b), larger diameter BNNTs appear to separate from each other in the entangled structures while smaller diameter BNNTs tended to bundle more, similar to typical few-walled carbon nanotube (FWCNT) samples ${ }^{[36]}$ but less extensive than SWCNTs. ${ }^{[8]}$ Most BNNTs were highly crystalline and had straight wall structures with layer-layer distance of $0.33 \mathrm{~nm}$ (Figure 1c-h). With high resolution imaging, atomic hexagonal crystalline structure of the BNNT surface could be readily observed (Figure 1e,f,h), allowing the classification of the nanotube chirality as indicated in the schematic drawings in the insets of these figures. A majority of the BNNTs were found to be either zigzag $\left(\theta=30^{\circ}\right.$; e.g. Figure $1 \mathrm{e}$ and $\mathrm{h}$ right) or armchair (chiral angle $\theta=0^{\circ}$; e.g. Figure $1 \mathrm{f}$ and h left) nanotubes rather than chiral ones $\left(0^{\circ}<\theta<30^{\circ}\right)$ according to the orientation of the hexagonal patterns. This result was consistent with previous reports. ${ }^{[1-3,37-39]}$ The surfaces of most BNNTs were covered with amorphous materials, which might be associated with amorphous BN fragments formed during the production. These amorphous BN impurities might be viewed to be equivalent to the amorphous carbons in as-produced CNT samples. ${ }^{[9-11]}$

\subsection{Aqueous Ammonia Solution for BNNT Modification}

The modification of BNNTs with aqueous ammonia solution using bath sonication was a straightforward process. The as-produced nanotubes $(5 \mathrm{mg})$ were added into an aqueous ammonia solution $(0.3-10 \mathrm{wt} \%, 10 \mathrm{~mL})$ in a round-bottomed flask and sonicated for a certain period of time $(2-50 \mathrm{~h})$. The initially greyish white-colored cotton-like hydrophobic nanotube solid 

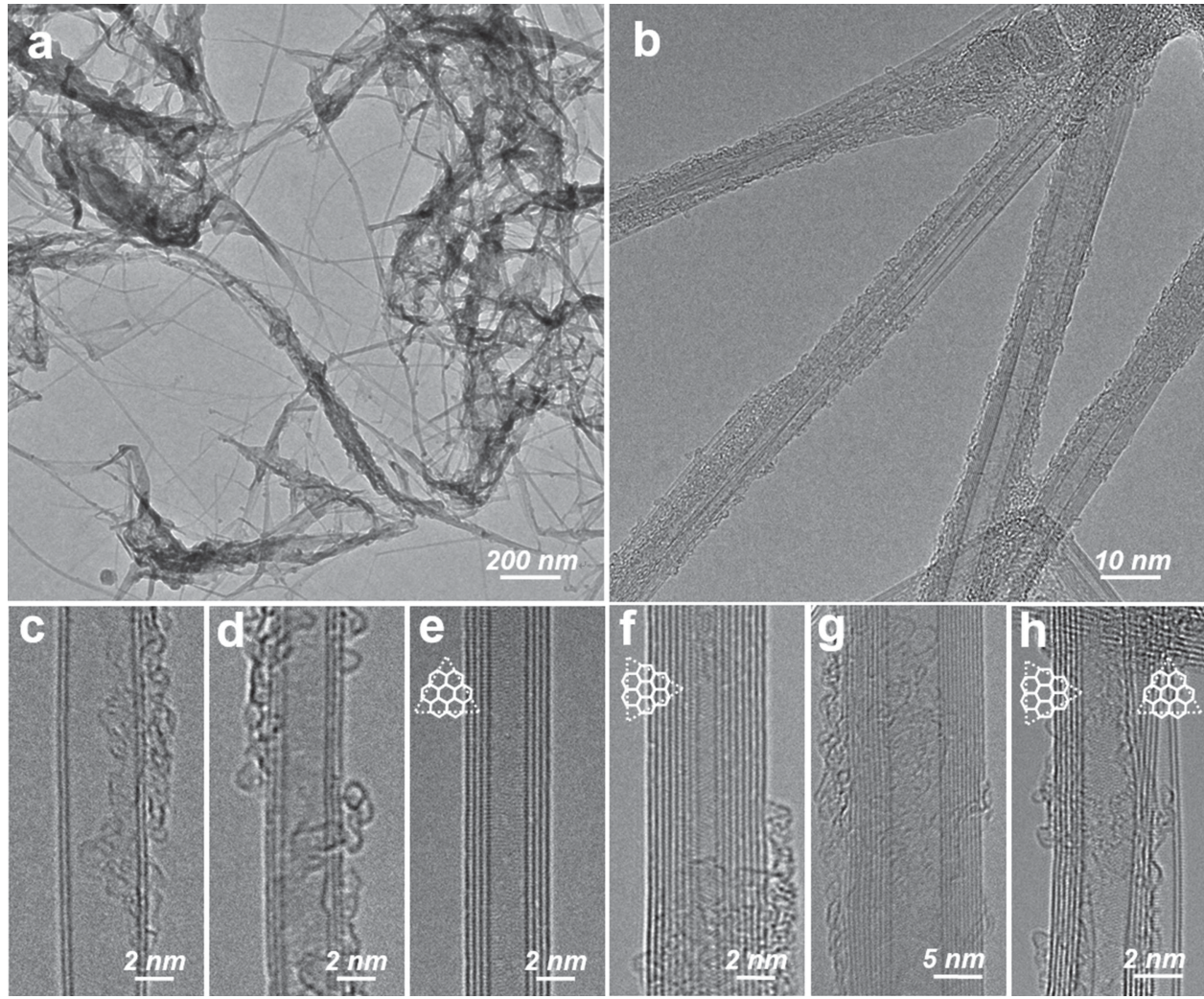

Figure 1. TEM images of the pristine BNNTs are shown at (a) lower and (b) higher magnifications. HR-TEM images show individual BNNTs of (c) two, (d) three, (e) four, ( $\mathrm{f}$ ) eight, and (g) eleven walls. Shown in (h) is a bundle of two BNNTs with six and two walls, respectively. Chirality patterns of some BNNTs under high-resolution were differentiated with appropriate imaging conditions. Each dot in the fringe pattern corresponds to a hexagon in the BNNT structure, thus the fringe patterns are the layouts of these hexagons. For example, the BNNTs in (e) and (h, tube on the right) are zigzag ones with fringe patterns either perpendicular or forming a $30^{\circ}$ angle with the nanotube sidewall. Those in (f) and ( $h$, tube on the left) are of armchair configuration with fringe patterns either parallel or forming a $60^{\circ}$ angle with the sidewall.

gradually dissociated and became dispersed in the solution. Upon centrifugation, the supernatant containing ammoniamodified BNNTs was collected as a milky yet transparent dispersion (Figure $2 \mathrm{a}$ inset, the vial on the right), similar to other functionalized $\mathrm{BNNT}^{[16-21]}$ or BNNS dispersions ${ }^{[40]}$ reported in the literature. Consistent with this appearance, the UV-vis optical absorption spectrum of the freshly obtained ammoniamodified BNNT dispersion was a featureless curve with a low intensity tail extending into the visible region (Figure 2a). Because of the overwhelming absorption cutoff at $<220 \mathrm{~nm}$ from the aqueous ammonia solution, the typical BN bandgap peak (often at $\sim 200 \mathrm{~nm}$ ) for the ammonia-modified BNNT sample could not be directly observed. However, the smooth featureless shape of the optical spectrum in the entire UV-visible range qualitatively indicated that there could be a broad distribution of bandgaps for the various BN species in the product due to chemical functionalization and corrosion (see next Section). Nevertheless, to monitor the progress of BNNT uptake in the dispersion during the treatment, we followed the sonication time-dependent evolution of the optical spectra of the supernatants separated from the reaction mixture, which were taken immediately after centrifugation. As shown in Figure $2 \mathrm{~b}$, the absorbance value of the dispersion at $350 \mathrm{~nm}$ reached a plateau after $\sim 24 \mathrm{~h}$ of sonication, indicating the saturation of BNNT uptake in the dispersion under the given conditions.
The use of aqueous ammonia solutions was proven critical to the significant dispersion uptake $(-25 \mathrm{wt} \%)$ of BNNTs. In a control experiment, the pristine BNNTs were directly sonicated in deionized water under the same conditions. Our previous studies showed that water may directly exfoliate and disperse BNNSs due to the combination of solvent polarity effect and sonication-assisted hydrolysis of hexagonal BN. ${ }^{[41]}$ Similarly, in this work, it was observed that the BNNTs, the rolled-up version of BNNS, could also be directly dispersed in water. However, the uptake of BNNTs in water was much less than in aqueous ammonia solutions. Although the weight balance for the waterbased uptake was too minute to be accurately measured, such a difference was visually implied for the as-prepared dispersions (Figure 2a inset) by their appearances (much less milky for direct water dispersion for the vial on the left) and the intensity of Tyndall phenomenon in which the laser beam passed through the dispersions (much less bright for direct water dispersion). More quantitatively, assuming that the optical absorption in near-UV region (e.g. $350-400 \mathrm{~nm}$ ) from ammonia is negligible and the BNNTs in both dispersions follow Lambert-Beer's law with similar extinction coefficients, the optical absorption spectra comparison (Figure 2a) suggests that the concentration of ammonia-modified BNNTs in $10 \%$ aqueous ammonia solution is roughly 8 times of the water-dispersed BNNTs. 

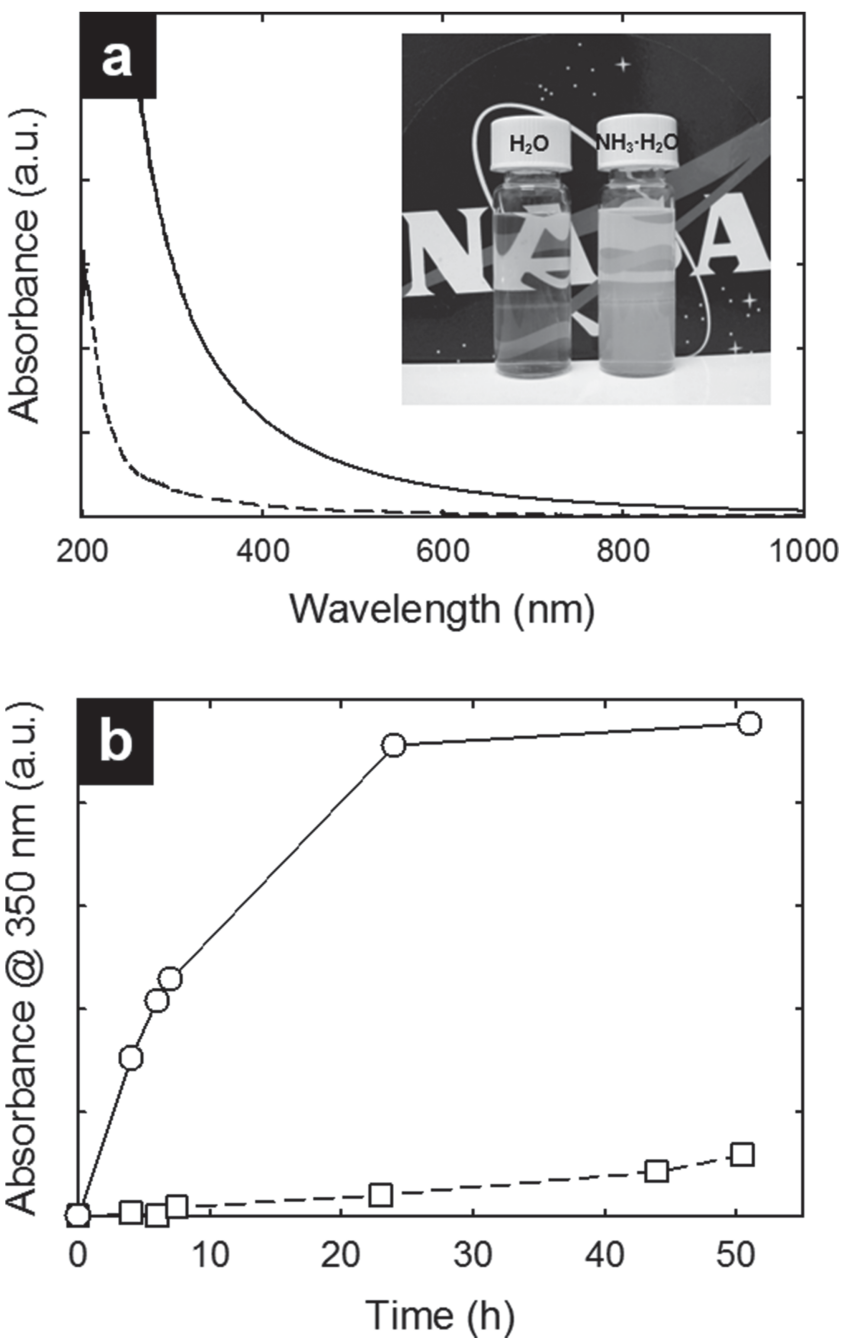

Figure 2. (a) Optical absorption spectra comparison of freshly prepared BNNT dispersion from sonication in $10 \%$ aqueous ammonia solution (solid) or deionized water (dashed) for $24 \mathrm{~h}$. The inset shows the corresponding photograph of the two fresh dispersions, with a laser beam entering from the left of the photograph to demonstrate the Tyndall effect; (b) The progresses of both reactions ( $10 \%$ aqueous ammonia solution: solid, open circle; water: dashed, open square) were followed by monitoring the optical absorption at $350 \mathrm{~nm}$ against the sonication time.

It has been established that the electron-deficient boron atoms on BNNT surfaces are susceptible to the attack of Lewis bases such as amines. ${ }^{[17-19]}$ As the smallest amine, ammonia should also be able to attach to the boron atoms via Lewis acidbase interactions. ${ }^{[42,43]}$ With high electron negativity of the nitrogen atom and small spatial molecular hindrance, there were abundant ammonia molecules attached to BNNTs, rendering the nanotubes with increased hydrophilicity and thus high dispersibility in water. In order to more quantitatively evaluate the amount of ammonia molecules attached, a BNNT sample treated with $10 \%$ aqueous ammonia solution for $24 \mathrm{~h}$ was carefully dried first in a steady stream of nitrogen followed by in vacuum at $\sim 60{ }^{\circ} \mathrm{C}$ overnight. The dried sample was then subjected to thermogravimetric analysis (TGA) in air at a heating rate of $10^{\circ} \mathrm{C} / \mathrm{min}$. As shown in Figure 3, the weight loss

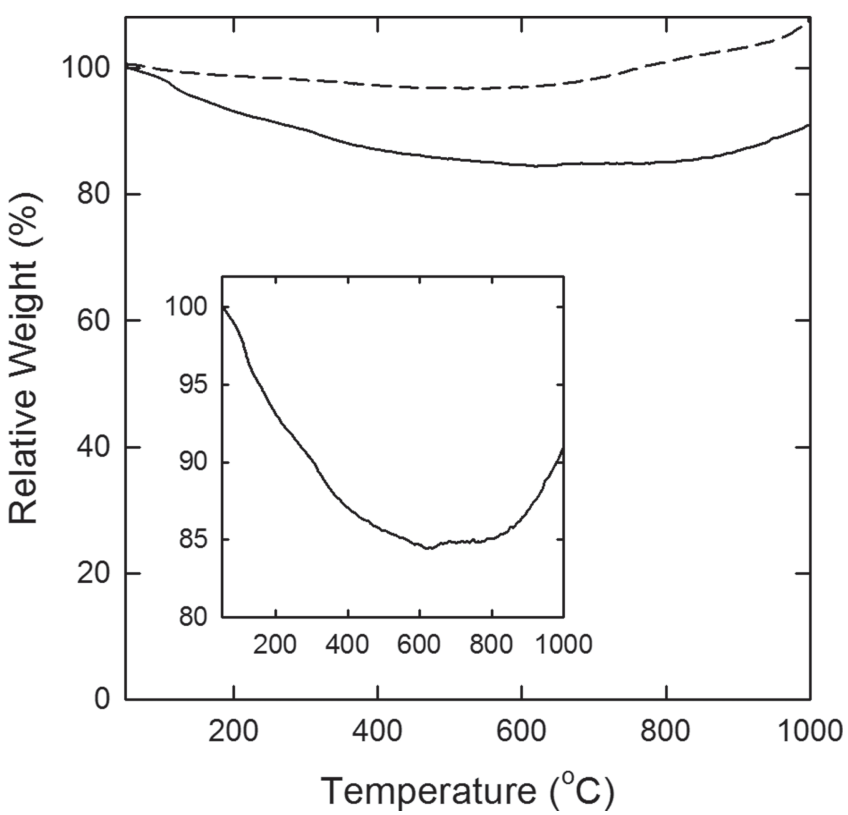

Figure 3. The TGA trace of a $10 \%$ ammonia-modified BNNT sample from $24 \mathrm{~h}$ sonication (solid) in comparison to that of the pristine BNNT sample (dashed). Inset is the same trace for the ammonia-modified BNNTs but with enlarged region in relative weight (y-axis).

of the ammonia-treated BNNT sample continuously progressed from temperatures below $100{ }^{\circ} \mathrm{C}$ to beyond $400{ }^{\circ} \mathrm{C}$, indicating a wide distribution of adsorption strengths of ammonia functionalities. The total weight loss of the sample measured at $600{ }^{\circ} \mathrm{C}$ was $\sim 15.6 \mathrm{wt} \%$, while the parent BNNT only showed a minor weight loss $(-3.8 \mathrm{wt} \%)$ at the same point. If such weight loss difference $(\sim 12 \mathrm{wt} \%)$ could be all attributed to ammonia functionalities, the data was equivalent to approximately 1 ammonia molecule per 4 to $5 \mathrm{~B}-\mathrm{N}$ bonds. However, this could be an overestimate as the resultant BNNTs could also contain some hydroxyl groups as a result from hydrolysis (see next section). The weight gain for both samples beyond $750{ }^{\circ} \mathrm{C}$ were typical for $\mathrm{BN}$ polymorphs due to their oxidation into $\mathrm{B}_{2} \mathrm{O}_{3} \cdot{ }^{[15]}$

Despite the abundant ammonia functionalities, the ammonia-modified BNNT dispersions exhibited significant agglomeration only after 1-2 days. It is likely that the small size of ammonia molecules were insufficient to prevent the interBNNT van der Waals interactions despite their strong attachment to the BNNT surfaces. As a result, the majority of characterizations were carried out on fresh samples.

Raman and FT-IR spectra were taken on the solid ammoniamodified BNNT products by similarly evaporating the solvent from a freshly prepared dispersion. As shown in Figure 4a, the Raman spectrum of an ammonia-modified BNNT sample exhibited the typical $E_{2 \mathrm{~g}}$ peak at $1360 \mathrm{~cm}^{-1}$, similar to that from the pristine BNNT sample. ${ }^{[1-3]}$ The peak did appear somewhat broadened, perhaps a consequence from the significant corrosive effects as discussed in the next section. The FT-IR spectrum of a similar sample (Figure $4 \mathrm{~b}$ ) exhibited distinct typical B-N bond network peaks at 1383 and $812 \mathrm{~cm}^{-1}$ inherited from parent BNNTs, which correspond to the in-plane $\mathrm{B}-\mathrm{N}$ transverse optical mode and the $\mathrm{B}-\mathrm{N}-\mathrm{B}$ out-of-plane bending vibrations, respectively. ${ }^{[1-3,40,44]}$ The broad peak centered at $\sim 3300 \mathrm{~cm}^{-1}$ was likely from $\mathrm{N}-\mathrm{H}$ 

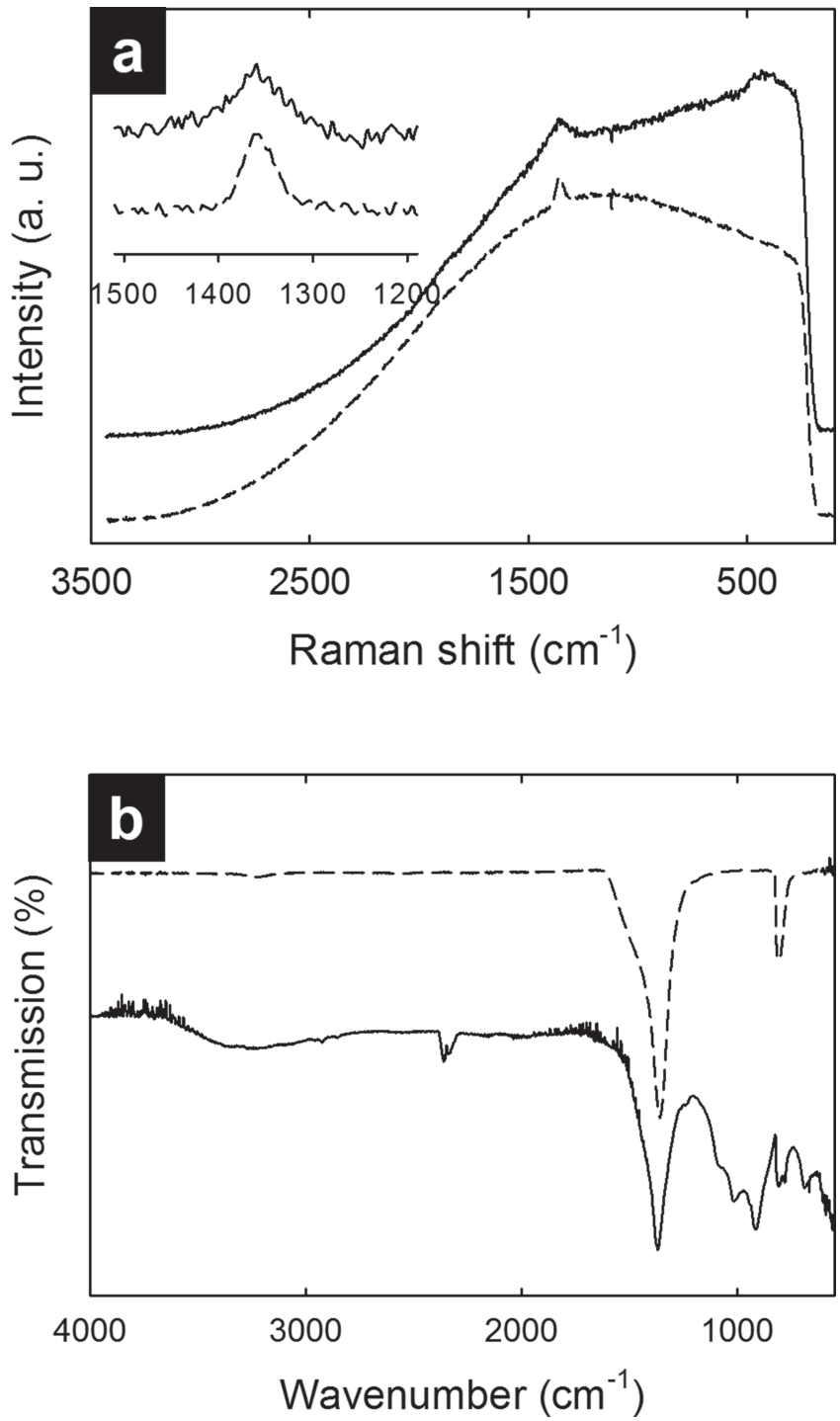

Figure 4. (a) Raman and (b) FT-IR spectra of a 10\% ammonia-modified BNNT sample with $24 \mathrm{~h}$ sonication (solid) and the pristine BNNTs (dashed).

stretching, which may be readily attributed to ammonia functionalities. A broad distribution of peaks appeared in the region of $1200-830 \mathrm{~cm}^{-1}$ (peaks at 1085, 1020, 945, 918, 781, $690 \mathrm{~cm}^{-1}$ ). Most peaks could be assigned to $\mathrm{B}-\mathrm{O}$ vibrations of various neighboring bond configurations ${ }^{[45]}$ as a result from the hydrolysis of the B-N bond network, which caused the significant BNNT corrosion that is discussed in more detail in the next section. In addition, one or more of these peaks near $811 \mathrm{~cm}^{-1}$ (the B-N-B bending vibration of the nanotube), such as the peak at $781 \mathrm{~cm}^{-1}$, might be attributed to similar vibration modes of $\mathrm{B}-\mathrm{N}$ bonds between boron atoms and the functionalized ammonia species.

\subsection{Corrosive Effects}

Detailed TEM investigations on the ammonia-modified BNNTs revealed significant corrosion of these presumably inert nanotubes. Compared to the pristine BNNTs with highly crystalline sidewalls (Figure 1) and intact end-caps (Figure 5a-c), the ammonia-modified BNNTs, even with only $2 \mathrm{~h}$ sonication using $3 \%$ aqueous ammonia solution, showed significant wall corrosion and thinning near the nanotube tip, an effect that might be referred to as "sharpening" (Figure 5d,e). The end-caps of some nanotubes were completely removed so that the tubes became open-ended (Figure 5f). The sharpening effect and the end-cap removal are likely a direct result of higher reaction susceptibility of the nanotube tip, where the joints of the flattened B-N bond network (typically with four- or eight-membered rings for BN polymorphs) ${ }^{[1,2]}$ exhibits larger curvature and likely more structural strain and discontinuity. This effect is similar to what was observed for the oxidation and other covalent functionalization of CNTs, where the nanotube ends and tips (five- or seven-membered rings are more common) were also reacted preferentially to the conjugated nanotube sidewall. ${ }^{[6-8,46]}$

The corrosion became even more extensive with either longer sonication time ( $3 \%$ for $8 \mathrm{~h}$, Figure $5 \mathrm{~g}-\mathrm{i}$ ) or higher concentration of the aqueous ammonia solution $(10 \%$ for $2 \mathrm{~h}$, Figure $5 \mathrm{j}-1)$. End-cap opening and even sidewall thinning became more common. For example, the sidewall of the BNNT in Figure $5 \mathrm{~h}$ was significantly corroded, while the nanotube in Figure $5 i$ was left with mostly double-walled but only a short multi-walled ring attached to a small section of the external wall. In Figure 51, a bundle of three open-ended double-walled BNNTs happened to bend toward the viewer, resembling the earlier concept of "fullerene pipes" for open-ended CNTs. ${ }^{\left[{ }^{[8}\right.}$

In a recent report, gas-phase ammonia plasma was used to functionalize BNNTs, but no nanotube wall corrosion or end-cap removal was reported. ${ }^{[47]}$ In the experiments reported herein, it is likely that the presence of both ammonia and water was critical to the corrosion of BNNTs under sonication conditions. Mechanistically, the observed corrosive effect can be viewed as a synergistic result from boron-ammonia (Lewis acid-base) interactions and sonication-induced hydrolysis of the B-N bond network. More specifically, as discussed in the previous section, ammonia molecules attach to BNNT surfaces more readily than larger Lewis base molecules (such as amine molecules with long lipophilic or hydrophilic chains $)^{[17-19]}$ due to the higher electronegativity and the almost negligible steric hindrance of ammonia. Therefore, the ionic attachments of a large number of ammonia molecules to the boron atoms on the nanotube surface could have significantly weakened the strengths of the B-N bond network, making them much more susceptible to sonication-assisted hydrolysis. The hydrolysisinduced BNNT corrosion should have allowed the attachment of oxygen-containing functional groups (such as hydroxyl groups) to the boron atoms at the damaged sites, as indicated from the broad distribution of $\mathrm{B}-\mathrm{O}$ vibration peaks in the fingerprint region of FT-IR spectrum (Figure 4b). Consistently, without the presence of ammonia, as in the case of the control experiment where only water was present, end cap removal or sidewall eroding/thinning was found to be much less even with much longer sonication time (although still present due to sonication/ hydrolysis). The use of sonication was also proven critical for the functionalization/corrosion to occur, since direct reflux of BNNTs in the same concentrations of ammonia solutions was not even able to wet the entire cotton-like pristine solid. 

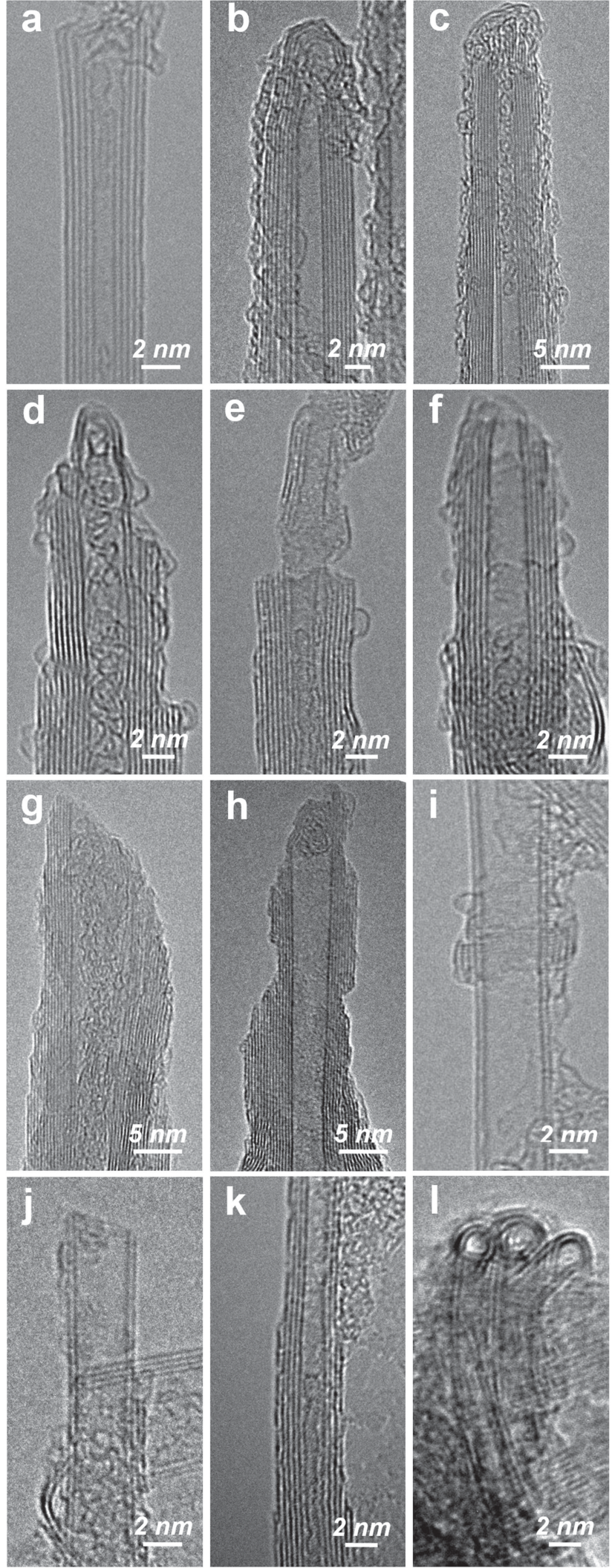

Figure 5. HR-TEM images of $(\mathrm{a}-\mathrm{c})$ pristine BNNTs, and also various modified BNNTs from sonication in (d-f) $3 \%$ aqueous ammonia solution for $2 \mathrm{~h}$, (g-i) $3 \%$ for $8 \mathrm{~h}$, and $(\mathrm{j}-\mathrm{l}) \mathrm{10} \%$ for $2 \mathrm{~h}$.
As also suggested from Figure 5, after the treatment with aqueous ammonia solutions, many nanotube surfaces appeared "cleaner" with much less amorphous material-likely amorphous BN fragments-commonly seen on the pristine nanotubes. This is understandable since, if BNNTs are reactive under the experimental conditions, amorphous BN materials should be more so. This result indicated that using aqueous ammonia solutions under appropriate conditions might be useful in the purification of as-produced BNNTs against amorphous BN impurities. Such characteristics of the corrosion effects of aqueous ammonia solutions on BNNTs resembles those of nitric acid (and other strong oxidative treatments) on CNTs. ${ }^{[10,11]}$ Both types of chemical treatments result in sharpening, end-cap removal, and sidewall thinning of the respective nanotubes, and are useful in the nanotube purification by removing respective amorphous impurities from the nanotube surface. Different from the oxidative nature of the nitric acidbased CNT treatments, against which BNNTs are known to be inert, the key for the efficiency of aqueous ammonia solutions on BNNTs lies in the combination of functionalization-induced $\mathrm{B}-\mathrm{N}$ bond tempering and the sonication-assisted hydrolysis.

The corrosive effects were further accompanied by the circumferential cutting or shortening of the BNNTs, which is also similar to the oxidative acid-induced CNT shortening. ${ }^{[8]}$ As shown in Figure $6 c$, the modified BNNTs in the centrifuged supernatant dispersion obtained after $24 \mathrm{~h}$ sonication in $10 \%$ aqueous ammonia solution (consisting of $\sim 25 \mathrm{wt} \%$ of total nanotube used) have an average length of $139 \mathrm{~nm}$, in comparison to the starting entangled nanotubes which were approximately tens of $\mu \mathrm{m}$ long. More detailed investigations revealed that the BNNTs were shortened progressively with sonication time. For example, the average lengths of the dispersed nanotubes obtained from 2 and $8 \mathrm{~h}$ sonication in the same aqueous ammonia solution were 283 and $187 \mathrm{~nm}$, respectively (Figure 6a,b). In addition, the concentration of the aqueous ammonia solution also affected the final BNNT length. For example, sonication of pristine BNNTs in 3\% aqueous ammonia solution for $24 \mathrm{~h}$ resulted in shortened nanotubes of $204 \mathrm{~nm}$ in average length.

Importantly, during the treatment with aqueous ammonia solutions, many BNNTs were also simultaneously longitudinally cut (or "unzipped"). Partially or fully unzipped nanotubes, the latter of which were essentially BNNRs, were observed in all aqueous ammonia solution-treated samples but absent in both the pristine BNNT sample and the control sample where only water was used in the sonication. Under lower magnification TEM, the partially and fully unzipped nanotubes, because of their flattened structure, exhibited larger width but much less contrast than the intact nanotubes of similar diameters. This contrast difference made the identification of BNNRs and partially unzipped BNNTs possible even when these nanostructures were on the carbon support of the TEM grid where the image contrast was too poor to enable unambiguous HR-TEM identification (vide infra). It should be noted that the starting BNNTs did contain a small fraction of BNNS impurities from production (Figure 1a). In comparison, the nanoribbons from unzipping exhibited higher aspect ratios and could thus be differentiated from the nanosheet impurities. For example, the arrows in Figure $6 \mathrm{a} \& \mathrm{~b}$ point to several identifiable partially 

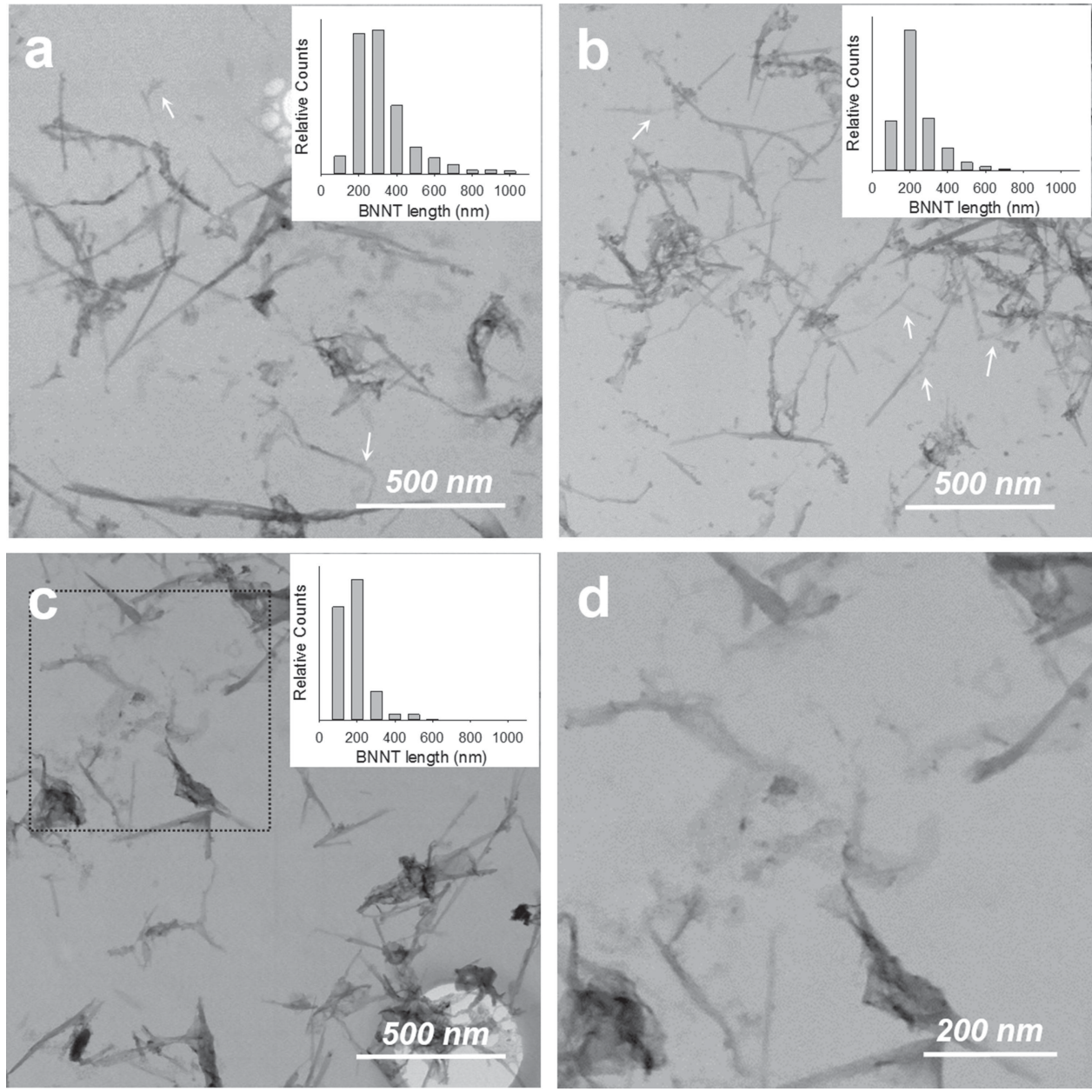

Figure 6. Low-magnification TEM images of $10 \%$ ammonia-modified BNNTs from sonication for (a) 2, (b) 8, and (c) $24 \mathrm{~h}$. Shown in the insets are the corresponding length distribution plots. Arrows in (a) and (b) point to a few partially unzipped nanotubes. (d) is an enlarged view of the highlighted area in (c) which were enriched with BNNR structures.

unzipped BNNTs in samples sonicated in $10 \%$ aqueous ammonia solution for 2 and $8 \mathrm{~h}$, respectively. Similar to the shortening effect discussed above, the extent of unzipping also depended upon the intensity of treatment. Longer sonication time and higher ammonia concentration led to more BNNRs or BNNTs with more unzipped extent. For example, with $24 \mathrm{~h}$ treatment of $10 \%$ aqueous ammonia solution, the majority $(>80 \%)$ of the BNNTs in the supernatant dispersion, being shortened in length, were at least partially unzipped (Figure 6c). Figure 6d highlights an area enriched with several BNNRs that were likely from full BNNT unzipping. A rough estimation of BNNRs from fully unzipped BNNTs consisted of about a few percent $(<5 \%)$ of the total sample.

Under HR-TEM, a variety of morphologies for unzipped BNNTs were observed. Shown in Figure 7a is a representative of a partially unzipped BNNT. The thick nanotube wall was partially peeled open, with flat sheet exposed underneath. In Figures 7b, a significant part of the right side of the parent BNNT were removed, leaving a "half nanotube"-like BNNR that were positioned sideways toward the viewer. Unlike the starting BNNTs (Figure 1), this unzipped nanotube had only one set of parallel lines on one side, enough to allow the counting of the layer number (4 layers). Figure 7c shows a top view of a 2-layer BNNR of $\sim 3.5 \mathrm{~nm}$ in width, which appeared to be a rather straight strip lying flat but with partially folded edges at its bottom half in the viewing area.

Longer BNNRs tended to bend, suggesting their high flexibility. As shown in Figures 7d-f, each BNNR was bent and partially positioned vertically toward the viewer, allowing the direct imaging of each BNNR edge from a side-view (indicated by the arrows). The layer numbers for these BNNRs were counted to be 5, 5, and 4, respectively. The edges of the BNNRs highlighted with dashed lines in Figures $7 \mathrm{~d}$ and e were relatively straight and without folding. Straight edge structures for BNNRs are known to be important for exploiting their theoretically predicted electronic properties. ${ }^{40]}$ Unfortunately, most of the BNNR edges observed in the current work were not well defined, likely due to the highly reactive reaction medium.

The fully dissociated BNNRs observed on the carbon support of the TEM grids (e.g. Figure 6d) had lengths of up to a few hundred nm. However, these long BNNRs tended to stay with the carbon support and thus were not suitable for HR-TEM imaging. The BNNRs suitable for high-resolution imaging 

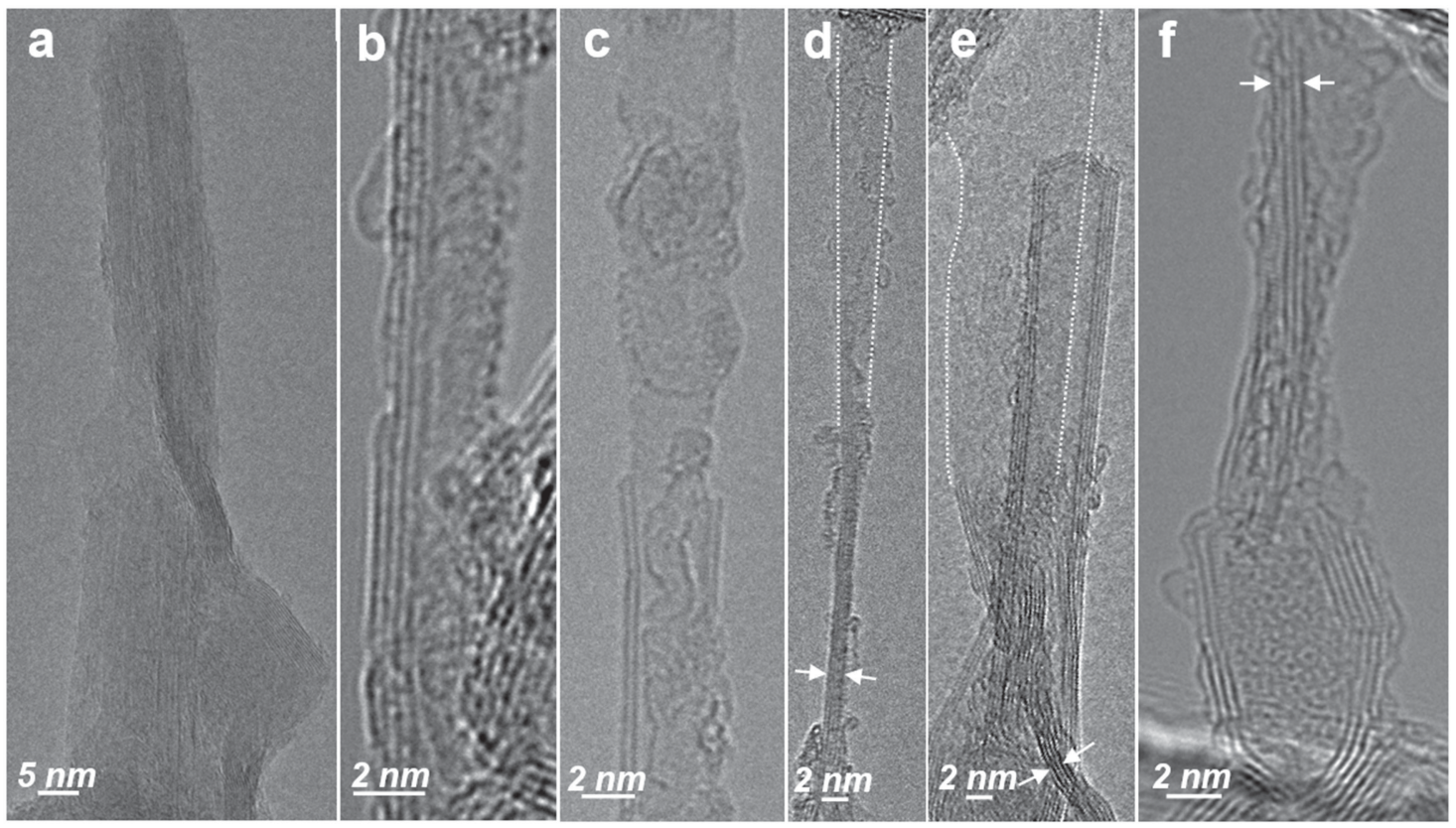

Figure 7. HR-TEM images of (a) a partially unzipped BNNT; (b) side view of a "half nanotube"-like BNNR; (c) top view of a fully unzipped BNNR with folded edges; and $(d-f)$ partially bent BNNRs (nearly full unzipping in the view area). The latter allowed the imaging of their edges from the side view. Dashed lines were added in (d) and (e) to highlight the low contrast ribbon edges. The red arrows indicate the side view of the ribbon edges.

typically had "exposed" lengths (i.e., the identifiable lengths across the carbon support holes) up to $\sim 100 \mathrm{~nm}$, with the rest of them entangled with other nanotubes or nanoribbons. The widths of most BNNRs were measured to be between -5-20 $\mathrm{nm}$. Considering the edge folding and the various corrosive effects, such widths were consistent with the diameter range of parent BNNTs $(\sim 2-10 \mathrm{~nm})$.

It is also important to characterize the chirality of BNNRs (zigzag or armchair) since the two configurations have different electronic properties. ${ }^{[40,48-50]}$ These properties are strongly related to their edge states. However, as discussed above, the edges of most BNNRs in this work were not quite well defined. Alternatively, the hexagon patterns on the surfaces of some BNNRs could be readily identified with available imaging resolution. By correlating these patterns with the length axis of the respective nanoribbons, the chirality information could be obtained. For example, as shown in the HR-TEM images in Figure 8, the zigzag directions were parallel to the length axis, indicating they were both of zigzag configurations. Armchair configurations from partially unzipped BNNTs were also found in the sample (not shown); however, the currently available data is not statistically sufficient to draw conclusions on the relative abundance of these two types. Nevertheless, thermodynamically, previous theoretical investigations have suggested that the bare zigzag edges of BNNRs are less stable than the armchair ones due to the existence of unpaired edge dangling bonds, and also have less stability as compared with the reconstructed edge made of 5-7-membered rings. ${ }^{[51-53]}$ After passivation with hydrogen atoms, however, the relative stabilities of both zigzag and armchair BNNRs became comparable. ${ }^{[51,52]}$ In this work, the BNNRs with rough edge structures were heavily functionalized with abundant ammonia molecules, which could also impart different stabilities of these two types of ribbons. A more detailed theoretical investigation is underway.

\section{Conclusion}

We have discovered a convenient method to disperse and chemically modify the morphology of BNNTs by sonication in aqueous ammonia solutions. While BNNTs have always been considered inert and are in need of highly reactive species to be covalently modified, the ammonia-modified BNNTs were significantly corroded and exhibited removed end-caps, sharpened ends, and thinned sidewalls, with the lengths much shortened compared to the starting nanotubes. These effects resemble the oxidative treatments of CNTs such as the use of nitric acid. With further optimization of the experimental conditions, this could lead to an effective purification technique for BNNTs by removing amorphous BN fragments from production. Importantly, many BNNTs were also partially or fully longitudinally cut, resulting in unzipped nanotubes, or BNNRs, that exhibited various morphologies. The products in the current investigation thus contained a mixture of various BN species, including openended/shortened BNNTs, fully or partially unzipped BNNRs, and other BN fragments. The corrosion of the ammonia solution also made the longitudinal edges of the BNNRs rough, similar to the corroded ends of the shortened/opened BNNTs. Future studies are underway to decouple these various corrosive effects, for instance, to selectively obtain shortened BNNTs or unzipped BNNRs with more ordered edges via different controlled chemical processes. It would be also helpful to apply the same method to BNNTs prepared with other methods ${ }^{[32-35]}$ since those nanotubes are of somewhat different structural morphology which may render interesting results. 

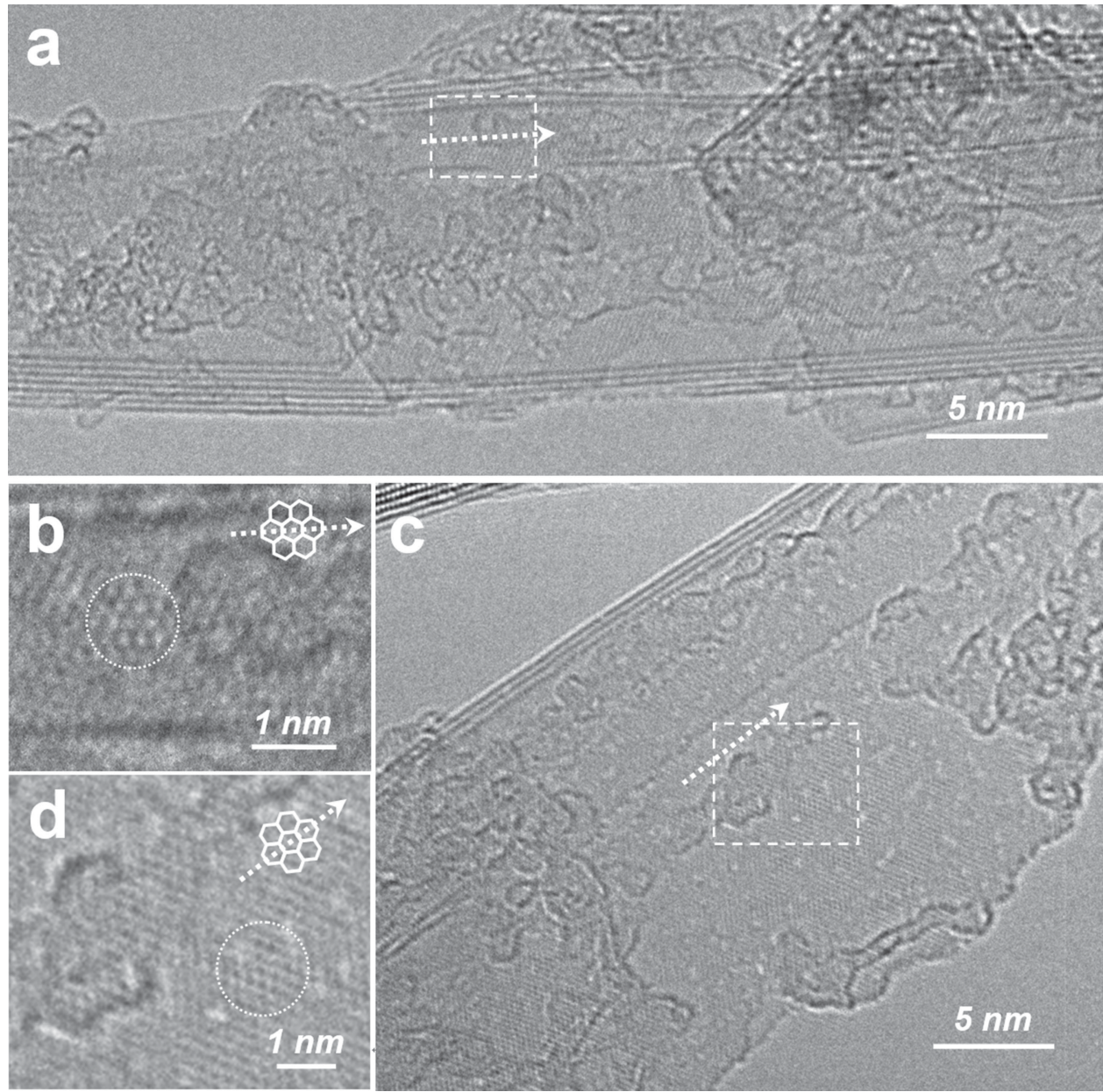

Figure 8. (a) and (c) are HR-TEM images of two BNNRs, which were identified by their lack of partial or full sidewall structure on one edge. (b) and (d) are the respective amplified views of the highlighted area in (a) and (c). The circles in (b) and (d) further highlight the hexagonal fringe patterns of the nanoribbons. The arrows point to the zigzag directions, which were parallel to the length axis of both nanoribbons, suggesting they were both of zigzag chirality.

\section{Experimental Section}

Materials: BNNTs were synthesized using the pressurized vapor condensation method with a $\mathrm{CO}_{2}$ laser as described previously. ${ }^{[30,31]}$ Aqueous ammonia solution (ammonium hydroxide, $30 \mathrm{wt} \%$, Certified ACS Plus) was purchased from Fisher Chemical.

Measurements: TEM imaging was carried out using a Hitachi S-5200 field-emission SEM system under the transmitted electron mode or a JEOL 2100 field-emission TEM system. The latter was also used for imaging under high-resolution (HR-TEM). TEM specimens were prepared by placing one or a few drops of the dispersion of interest onto a holey carbon-coated copper grid followed by solvent evaporation. For specimen preparation, aqueous ammonia-modified BNNT dispersions were directly used, while pristine BNNTs were dispersed in methanol. Optical absorbance spectra were obtained using a Perkin-Elmer Lambda 900 UV/ vis/NIR spectrometer. FT-IR spectra were obtained on a Thermo-Nicolet FT-IR 300 spectrometer equipped with a Thunderdome Swap-Top single reflection attenuated total reflectance (ATR) module. Raman spectroscopy was conducted using a Thermo-Nicolet-Almega dispersive Raman spectrometer with $785 \mathrm{~nm}$ excitation. TCA data was obtained at $10^{\circ} \mathrm{C} /$ min in air on a Netzsch TG $209 \mathrm{~F} 1$ Libra thermogravimetric analyzer.

Treatment of BNNTs with Aqueous Ammonia Solutions: In a typical procedure, a BNNT sample $(5 \mathrm{mg})$ was sonicated in an aqueous ammonia solution $(10 \%, 10 \mathrm{~mL})$ for $24 \mathrm{~h}$ using a bath sonicator (Branson $2510,40 \mathrm{kHz}$ ). The reaction flask was capped with a rubber stopper to avoid loss of volatile reagents. Upon completion of the sonication, the mixture was subjected to centrifugation at $4000 \mathrm{rpm}$ (Eppendorf 5810R) to separate the supernatant dispersion from the residue. The supernatant was then collected as the ammonia-modified BNNT dispersion. The concentration of the aqueous ammonia solution and the sonication time were both varied to obtain a series of ammonia-modified BNNT samples.

\section{Acknowledgements}

We thank Dr. W. Cao and Prof. H. Elsayed-Ali (Applied Research Center, Old Dominion University) for assistance in HR-TEM imaging and C. Chamberlain (NASA Langley Research Center) for TGA measurements. Y. Liao was an exchange student resident from University of Puerto Rico, who is supported by Department of Defense (Grant W911NF-121-0083) and NASA (Grant Nos. NNX10AM80H and NNX13AB22A) to conduct research at National Institute of Aerospace and NASA Langley Research Center. C. Park acknowledges the financial support in part by US Air Force Office of Scientific Research - Low Density Materials program (Grant No. FA9550-11-1-0042).

Received: February 20, 2014

Revised: March 7, 2014

Published online: April 22, 2014 
[1] D. Golberg, Y. Bando, C. C. Tang, C. Y. Zhi, Adv. Mater. 2007, 19, 2413.

[2] D. Golberg, Y. Bando, Y. Huang, T. Terao, M. Mitome, C. Tang, C. Zhi, ACS Nano 2010, 4, 2979.

[3] J. Wang, C. H. Lee, Y. K. Yap, Nanoscale 2010, 2, 2028.

[4] C. Zhi, Y. Bando, T. Terao, C. Tang, H. Kuwahara, D. Golberg, Adv. Funct. Mater. 2009, 19, 1857.

[5] G. Ciofani, V. Raffa, A. Menciassi, A. Cuschieri, Nano Today 2009, 4, 8.

[6] S. C. Tsang, P. J. F. Harris, M. L. H. Green, Nature 1993, 362, 520.

[7] D. Ugarte, A. Châtelain, W. A. de Heer, Science 1996, 274, 1897.

[8] J. Liu, A. G. Rinzler, H. Dai, J. H. Hafner, R. K. Bradley, P. J. Boul, A. Lu, T. Iverson, K. Shelimov, C. B. Huffman, F. Rodriguez-Macias, Y.-S. Shon, T. R. Lee, D. T. Colbert, R. E. Smalley, Science 1998, 280, 1253.

[9] A. C. Dillon, T. Gennett, K. M. Jones, J. L. Alleman, P. A. Parilla, M. J. Heben, Adv. Mater. 1999, 11, 1354.

[10] H. Hu, B. Zhao, M. E. Itkis, R. C. Haddon, J. Phys. Chem. B 2003, 107, 13838.

[11] R. C. Haddon, J. Sippel, A. G. Rinzler, F. Papadimitrakopoulos, MRS Bull. 2004, 29, 252.

[12] D. V. Kosynkin, A. L. Higginbotham, A. Sinitskii, J. R. Lomeda, A. Dimiev, B. K. Price, J. M. Tour, Nature 2009, 458, 872.

[13] A. L. Higginbotham, D. V. Kosynkin, A. Sinitskii, Z. Sun, J. M. Tour, ACS Nano 2010, 4, 2059.

[14] L. Jiao, X. Wang, G. Diankov, H. Wang, H. Dai, Nat. Nanotechnol. 2010, 5, 321.

[15] Y. Chen, J. Zou, S. J. Campbell, G. Le Caer, Appl. Phys. Lett. 2004, 84, 2430

[16] a)C. Zhi, N. Hanagata, Y. Bando, D. Golberg, Chem. Asian J. 2011, 6, 2530; b)C. Zhi, Y. Bando, T. Terao, C. C. Tang, H. Kuwahara, D. Golberg, Chem. Asian J. 2009, 4, 1536.

[17] S.-Y. Xie, W. Wang, K. A. S. Fernando, X. Wang, Y. Lin, Y.-P. Sun, Chem. Commun. 2005, 3670.

[18] S. Pal, S. R. C. Vivekchand, A. Govindaraj, C. N. R. Rao, J. Mater. Chem. 2007, 17, 450.

[19] A. Maguer, E. Leroy, L. Bresson, E. Doris, A. Loiseau, C. Mioskowski, J. Mater. Chem. 2009, 19, 1271.

[20] C. Zhi, Y. Bando, C. Tang, S. Honda, K. Sato, H. Kuwahara, D. Golberg, Angew. Chem. Int. Ed. 2005, 44, 7932.

[21] Z. Sheng-Jun, M. Chun-Yin, M. Ye-Yong, S. Hai-Feng, Z. Zheng, D. Shun-Liu, X. Su-Yuan, Nanotechnology 2012, 23, 055708.

[22] C. Zhi, Y. Bando, C. Tang, D. Golberg, Phys. Rev. B 2006, 74, 153413.

[23] T. Sainsbury, A. Satti, P. May, A. O'Neill, V. Nicolosi, Y. K. Gun'ko, J. N. Coleman, Chem. Eur. J. 2012, 18, 10808.

[24] T. Sainsbury, A. Satti, P. May, Z. Wang, I. McGovern, Y. K. Gun'ko, J. Coleman, J. Am. Chem. Soc. 2012, 134, 18758.

[25] C. H. Lee, D. Zhang, Y. K. Yap, J. Phys. Chem. C 2011, 116, 1798.
[26] H. Zeng, C. Zhi, Z. Zhang, X. Wei, X. Wang, W. Guo, Y. Bando, D. Golberg, Nano Lett. 2010, 10, 5049.

[27] K. J. Erickson, A. L. Gibb, A. Sinitskii, M. Rousseas, N. Alem, J. M. Tour, A. K. Zettl, Nano Lett. 2011, 11, 3221.

[28] L. Jiao, L. Zhang, X. Wang, G. Diankov, H. Dai, Nature 2009, 458, 877.

[29] D. V. Kosynkin, W. Lu, A. Sinitskii, G. Pera, Z. Sun, J. M. Tour, ACS Nano 2011, 5, 968.

[30] M. W. Smith, K. C. Jordan, C. Park, J.-W. Kim, P. T. Lillehei, R. Crooks, J. S. Harrison, Nanotechnology 2009, 20, 505604.

[31] M. Zheng, C. Ke, I.-T. Bae, C. Park, M. W. Smith, K. Jordan, Nanotechnology 2012, 23, 095703.

[32] C. Zhi, Y. Bando, C. Tang, D. Golberg, Solid State Commun. 2005, $135,67$.

[33] C. H. Lee, M. Xie, V. Kayastha, J. S. Wang, Y. K. Yap, Chem. Mater. 2010, 22, 1782.

[34] W.-Q. Han, P. J. Todd, M. Strongin, Appl. Phys. Lett. 2006, 89, 173103.

[35] Y. Chen, J. F. Gerald, J. S. Williams, S. Bulcock, Chem. Phys. Lett. 1999, 299, 260.

[36] Y. Hou, J. Tang, H. Zhang, C. Qian, Y. Feng, J. Liu, ACS Nano 2009, 3, 1057.

[37] A. Loiseau, F. Willaime, N. Demoncy, G. Hug, H. Pascard, Phys. Rev. Lett. 1996, 76, 4737.

[38] M. Menon, D. Srivastava, Chem. Phys. Lett. 1999, 307, 407.

[39] R. Arenal, M. Kociak, A. Loiseau, D. J. Miller, Appl. Phys. Lett. 2006, 89, 073104.

[40] a)C. Zhi, Y. Bando, C. Tang, H. Kuwahara, D. Golberg, Adv. Mater 2009, 21, 2889; b)Y. Lin, T. V. Williams, J. W. Connell, J. Phys. Chem. Lett. 2010, 1, 277; c)Y. Lin, J. W. Connell, Nanoscale 2012, 4, 6908.

[41] Y. Lin, T. V. Williams, T.-B. Xu, W. Cao, H. E. Elsayed-Ali, J. W. Connell, J. Phys. Chem. C 2011, 115, 2679.

[42] X. Wu, W. An, X. C. Zeng, J. Am. Chem. Soc. 2006, 128, 12001.

[43] Y. Li, Z. Zhou, J. Zhao, J. Chem. Phys. 2007, 127, 184705.

[44] C. Zhi, Y. Bando, C. Tang, D. Golberg, R. Xie, T. Sekigushi, Appl. Phys. Lett. 2005, 86, 213110.

[45] W.-Q. Han, H.-G. Yu, C. Zhi, J. Wang, Z. Liu, T. Sekiguchi, Y. Bando, Nano Lett. 2008, 8, 491

[46] Z. Chen, W. Thiel, A. Hirsch, ChemPhysChem 2003, 4, 93.

[47] T. Ikuno, T. Sainsbury, D. Okawa, J. M. J. Fréchet, A. Zettl, Solid State Commun. 2007, 142, 643

[48] A. J. Du, S. C. Smith, G. Q. Lu, Chem. Phys. Lett. 2007, 447, 181.

[49] Z. Zhang, W. Guo, Phys. Rev. B 2008, 77, 075403.

[50] C.-H. Park, S. G. Louie, Nano Lett. 2008, 8, 2200.

[51] R. Mukherjee, S. Bhowmick, J. Chem. Theory Comput. 2011, 7, 720.

[52] B. Huang, H. Lee, B.-L. Gu, F. Liu, W. Duan, Nano Res. 2012, 5, 62

[53] M. Wu, X. Wu, Y. Pei, X. Zeng, Nano Res. 2011, 4, 233. 